^ VIBEYOR

\title{
A Short Review on Mechanical Behavior of Nanocrystalline Materials
}

\author{
Liang Tian*
}

Department of Materials Science and Engineering, lowa State University, USA

\begin{abstract}
Nanocrystalline materials, as a new generation of advance materials, have outstanding mechanical properties such as high strength and hardness, low elastic modulus, good ductility and excellent fatigue and wear resistance. In this paper, an overview of the synthesis and mechanical properties of nanocrystalline materials is provided. Furthermore, the grain size dependent plastic deformation mechanism is discussed to correlate with their mechanical behavior.
\end{abstract}

\section{Keywords}

Nanocrystalline materials, Mechanical properties, Deformation mechanism

\section{Introduction}

Nanocrystalline materials are ultrafine-grained polycrystals with a grain size under $100 \mathrm{~nm}$. In the order of a few nanometers, these materials consist of about $50 \mathrm{vol}$. $\%$ crystals (crystalline component) and 50 vol. \% interfaces (interfacial component) [1]. The concept of nanocrystalline materials was first introduced by Gleiter in 1986 [1]. He found that nanocrystalline materials can allow the alloying of components which are immiscible in conventional coarse-grained materials and the properties of nanocrystalline materials are different from those of single crystals, coarse-grained polycrystals and glasses with the same average chemical composition. He thinks that this deviation is strongly related to the reduced size of the crystallites as well as the large amount of grain boundaries between adjacent crystallites [1-6].

Nanocrystalline materials have been a hot research topic over the past 30 years since Gleiter presented the concept [3-25]. Figure 1a shows that a two-dimensional model of a nanocrystalline materials. Figure $1 \mathrm{~b}$ gives the effect of grain size on the volume fraction of intercrystal regions and triple junctions. The volume fraction of interfaces can be as much as $50 \%$ for $5 \mathrm{~nm}$ grains, $30 \%$ for $10 \mathrm{~nm}$ grains. Due to the small grain size, nanocrystalline materials often have superior properties to those of conventional polycrystalline coarse-grained materials. They exhibit increased strength, hardness, ductility, toughness, and reduced elastic modulus. Driven by this strong incentive, many research studies have been done to understand processing, structure, property relationship of nanocrystalline materials [21-29].

The synthesis and use of nanostructures can be at least traced back to 1976. Lashmore, et al. [25] found that the microstructure of polycrystalline iron whiskers is composed of a center core surrounded by many layers of concentric cylinders with particle diameter between 100 and $300 \mathrm{~nm}$. The grain size in both the core and the surrounding particles is between 5 and $20 \mathrm{~nm}$. The very high strength of these whiskers, $800 \mathrm{MPa}$, was observed. They believed that the extreme grain refinement is the strengthening mechanism for these whiskers. A schematic drawing of a cross section through a whisker is given in Figure 2.

Therefore, it is natural to think that the grain size distribution and the grain boundary structure can contribute to the mechanical behavior and deformation mechanisms of nanocrystalline materials [4]. Nieman, et al. [26] studied the bulk mechanical properties of nano-

${ }^{*}$ Corresponding author: Liang Tian, Department of Materials Science and Engineering, lowa State University, 2200 Bonisteel, Ann Arbor, MI, 48109, USA, Tel: +1-515-509-0857, E-mail: liangtianisu@gmail.com/lilangt@umich.edu

Received: June 26, 2017: Accepted: September 25, 2017: Published: October 13, 2017

Copyright: @ 2017 Tian L. This is an open-access article distributed under the terms of the Creative Commons Attribution License, which permits unrestricted use, distribution, and reproduction in any medium, provided the original author and source are credited. 

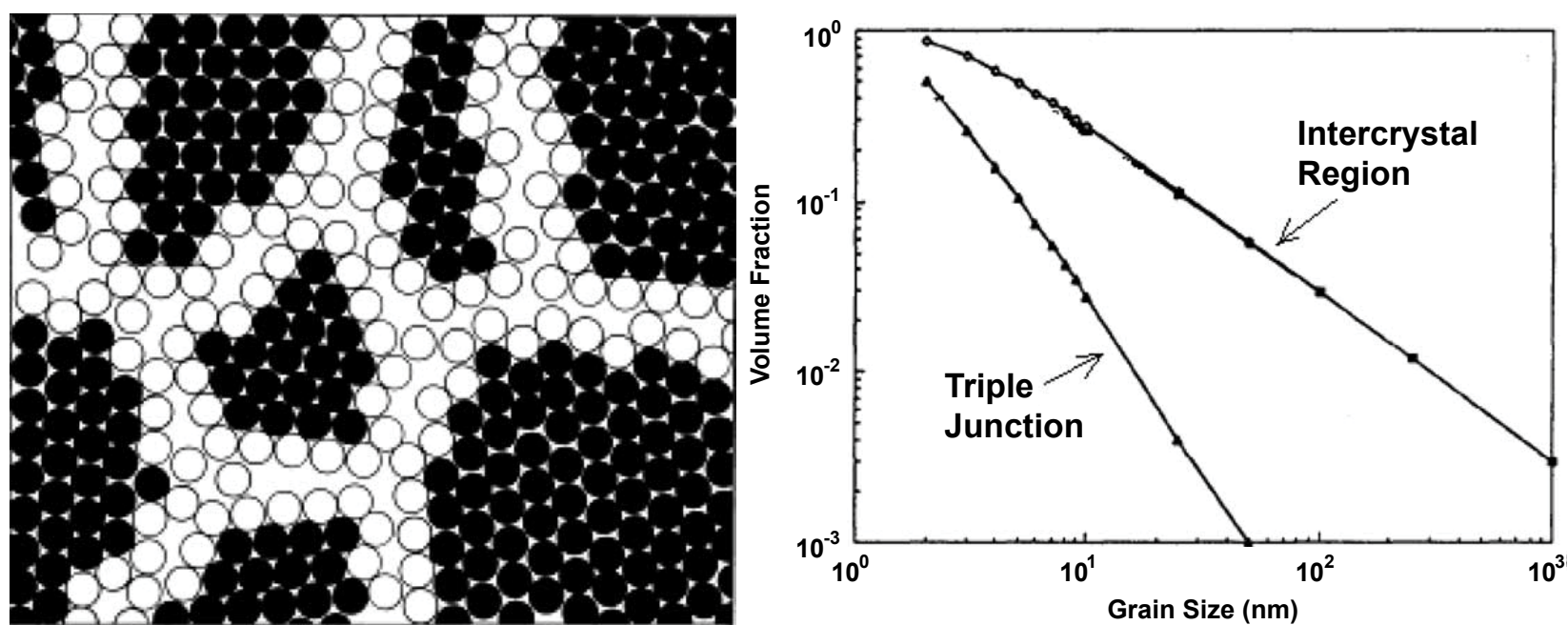

Figure 1: a) Two dimensional model of a nanostructured material; b) The effect of grain size on volume fraction of intercrystal regions and triple junctions (Adapted from Ref [5]).

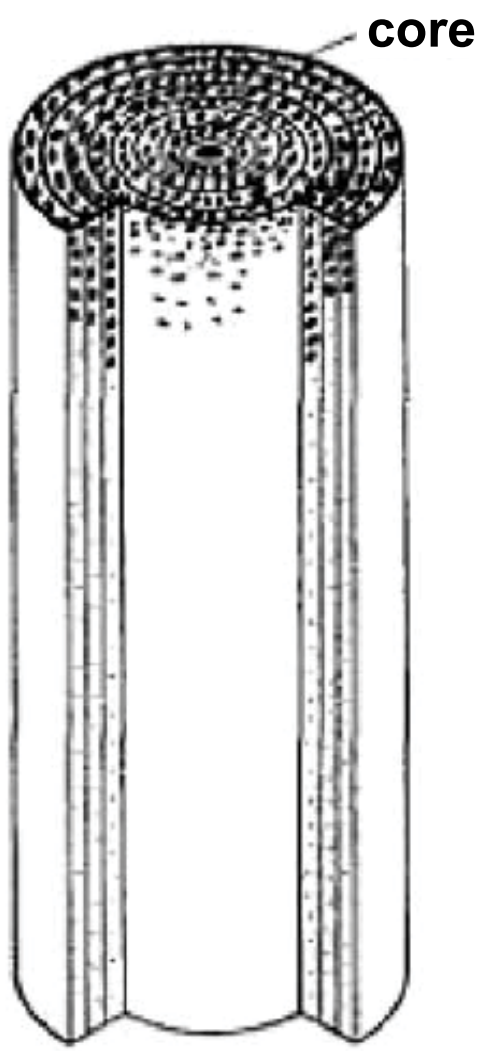

Figure 2: A schematic drawing of a cross section of a whisker. It shows a series of concentric cylindrical shells made up of individual particles (Adapted from Ref [25]).

crystalline $\mathrm{Cu}$ and $\mathrm{Pd}$ sample, which is prepared by inert gas condensation. The results showed that for nanocrystalline sample, the grain size is in the range of 3-50 nm, lattice strain is in the range of $0.02-3 \%$, and density is in the range of $97-72 \%$ of a coarse-grained sample. Microhardness and tensile strength of nanocrystalline samples exceeds that of annealed, coarse-grained samples. The author believed that the strengthening mechanism is the restrictions on dislocation generation and mobility due to ultrafine grain size. Fougere, et al. [28] prepared nanocrystalline Fe samples by inert gas condensation. A new consolidation device was used to minimize defect population and contamination. The Vickers microhardness of nanocrystalline Fe with grain size from 4 to $15 \mathrm{~nm}$ is 3 to 7 times that of coarse-grained Fe.

In addition, Sergueeva, et al. [21] demonstrated the role of grain boundary sliding and grain rotation in the deformation of nanocrystalline materials by forming homogeneous nanocrystalline NiTi by nanocrystallization of amorphous NiTi under high pressure torsion. The resultant nanostructure has $2650 \mathrm{MPa}$ room temperature strength with an elongation to failure of about $5 \%$. At elevated temperatures around $0.4-0.5 \mathrm{Tm}$, nanocrystalline NiTi showed a high ultimate strength with sufficient elongation (up to 200\%). Considering the shape and the size of grains did not change too much after deformation, they believed that in nanocrystalline $\mathrm{NiTi}$, grain boundary sliding and grain rotation are active and the generation and motion of dislocations play the role of accommodation of stress concentration.

The simultaneous high strength and ductility of nanocrystalline material have been demonstrated by many researchers. Valiev, et al. [22] proved that in the nanostructured titanium processed by severe plastic deformation, the controlled annealing could enhance both strength and ductility. Youssef, et al. [24] made artifact-free bulk nanocrystalline copper samples with a mean grain size $23 \mathrm{~nm}$. The material exhibited tensile yield strength about 11 times higher than that of conventional coarse-grained copper, while retaining a $14 \%$ uniform tensile elongation. Sergueeva, et al. [27] investigated the room temperature mechanical properties of commercially pure Ti with grain size from $60-150 \mathrm{~nm}$ by severe plastic deformation with annealing. The ultimate tensile strength significantly increased to $1150 \mathrm{MPa}$ with an elongation $8 \%$. 
Youssef, et al. [24] demonstrated that the dislocation based deformation mechanism is a major deformation mechanism in nanocrystalline copper by observing individual dislocation motion and dislocation pile-ups using in situ dynamic strain TEM.

Carsley, et al. [23] demonstrated the unique mechanical behavior of a bulk nanostructure $\mathrm{Fe}-10 \mathrm{Cu}$ alloy with grain sizes between $30 \mathrm{~nm}$ and $2 \mathrm{~mm}$. The only plastic deformation mechanism was shear banding, which was then accompanied by a perfectly plastic stress-strain response without strain hardening effect at temperature range from 77 to $470 \mathrm{~K}$, which resemble the mechanical behavior of amorphous materials.

Agnew, et al. [29] investigated the effects of inherent defects in nanocrystalline $\mathrm{Cu}$ and $\mathrm{Ni}$ on their mechanical behavior. The results showed that flaws, pores, and partial recrystallization determine the strength and may introduce some plasticity into the nanocrystalline samples.

Nanocrystalline materials are a subset of nanostructured materials, which include any material with one or more dimensions in the nanometer range [23]. Based on dimensionality, there are four categories of nanostructured materials: 0D-nanoparticles; 1D-nanowires, nanotubes, nanorods; 2D-nanograined layers; 3D-equilaxed bulk solids [5]. In this paper, nanocrystalline materials mean 3D bulk nanostructured materials. In the following sections, we will review the synthesis and mechanical behavior of nanocrystalline materials. Then discussion will be focused on grain size dependent plastic deformation mechanisms.

\section{Synthesis}

Two ideas to synthesize nanocrystalline materials is either consolidating small clusters or breaking down the coarse-grained polycrystalline bulk material into nano-grained crystallites [5]. Based on this, the principle synthesis methods can be generally classified into four groups: inert gas condensation, mechanical alloying and compaction, severe plastic deformation and electrodeposition [4].

Mechanical alloying, essentially ball milling in a protective atmosphere, has been used to produce nanostructured materials via repeated grinding. Nano-sized grains can be obtained after sufficient milling time. The grain growth is not significant during consolidation of this process, which makes the grain size easy to control. The advantage of this process is to produce nanostructured materials in reasonable size and quantities. Its disadvantages include the difficulty to control materials purity and achieving full density. The method was particularly successful for aluminum alloys. The relative mechanical behavior data and the associated cryomilling mechanisms for formation of nanostructured $\mathrm{Al}$ alloys are given in reference [30]. Koch [11] has reviewed the synthesis and structure of nanocrystalline materials by this technique. Other examples using this technique can be seen in references [30-34].

Inert gas condensation has usually been used to produce nanocrystalline $\mathrm{Cu}, \mathrm{Ni}, \mathrm{Pd}$ by evaporating a metal inside a chamber with high vacuum and then backfilling a low pressure inert gas $[24,26,35,36]$. In reference $[28,37]$, inert has condensation has also been used to produce nanocrystalline $\mathrm{Fe}$ and TiAl alloys. As an early technique to produce ultrafine grain materials, the use of this technique can be traced back to 1981 [38]. Muhlbach, et al. [37] used inert gas condensation to produce microclusters of metal atoms $\mathrm{Sb}, \mathrm{Bi}$ and $\mathrm{Pb}$. They proved that condensation parameters (e.g. type of inert gas, temperature of the inert gas, temperature of the metal oven) can influence the grain size distribution. The principle of inert gas condensation is that the evaporated metal atoms collide with the gas atoms, lose the kinetic energy, and condense into small particles. Then the condensed fine particles were carried by the convection currents to the collector device. Then a compaction process is taken to produce the final bulk nanocrystalline materials. Smaller grain size can be obtained by decreasing either inert gas pressure or the evaporation rate and by using light weight inert gases. The limitations of this technique include impurity in the vicinity of the particle boundaries, porosity due to insufficient consolidation, difficulties to maintain the fine grain size during consolidation. Sanders, et al. [39] has reported to use new synthesis and compaction devices to reduce hydrogen and oxygen impurities and increase density. However, they found that it was very difficult to produce and retain extremely fine grain sizes in the high purity and high density nanocrystalline $\mathrm{Pd}$ and $\mathrm{Cu}$ synthesized in this study. Markmann, et al. [40] used the cold rolling to increase the density of the specimens while still retaining the size of the texture-free, equiaxed grains.

Electrodeposition has been used to produce sheets of nanocrystalline metals (e.g. $\mathrm{Ni}, \mathrm{Cu}, \mathrm{Zn}, \mathrm{Ag}$ ) [41-47] and binary alloys (e.g. Ni-Fe, Ni-W, Ni-Cu) [48-51]. The advantages of this technique include obtaining porosity-free products, no need for consolidation process, low investment and high product rates. Pulse electrodeposition technique exhibits obvious advantages over direct current electrodeposition in the control of deposit grain size, surface morphology, and preferred orientation [45,46]. It has been shown that the electrodeposition parameters can influence the final grain size and should be properly chosen to produce nano-size grains by favoring the nucleation of new grains instead of growth of existing grains $[5,41,43]$. This can be done by using high deposition rates, formation of appropriate complexes in bath, addition of suitable surface-active elements to reduce surface diffusion $[5,43]$. $\mathrm{Qu}$, et al. [43] developed a new method to synthesize bulk nanocrystalline nickel without using any additives. They 

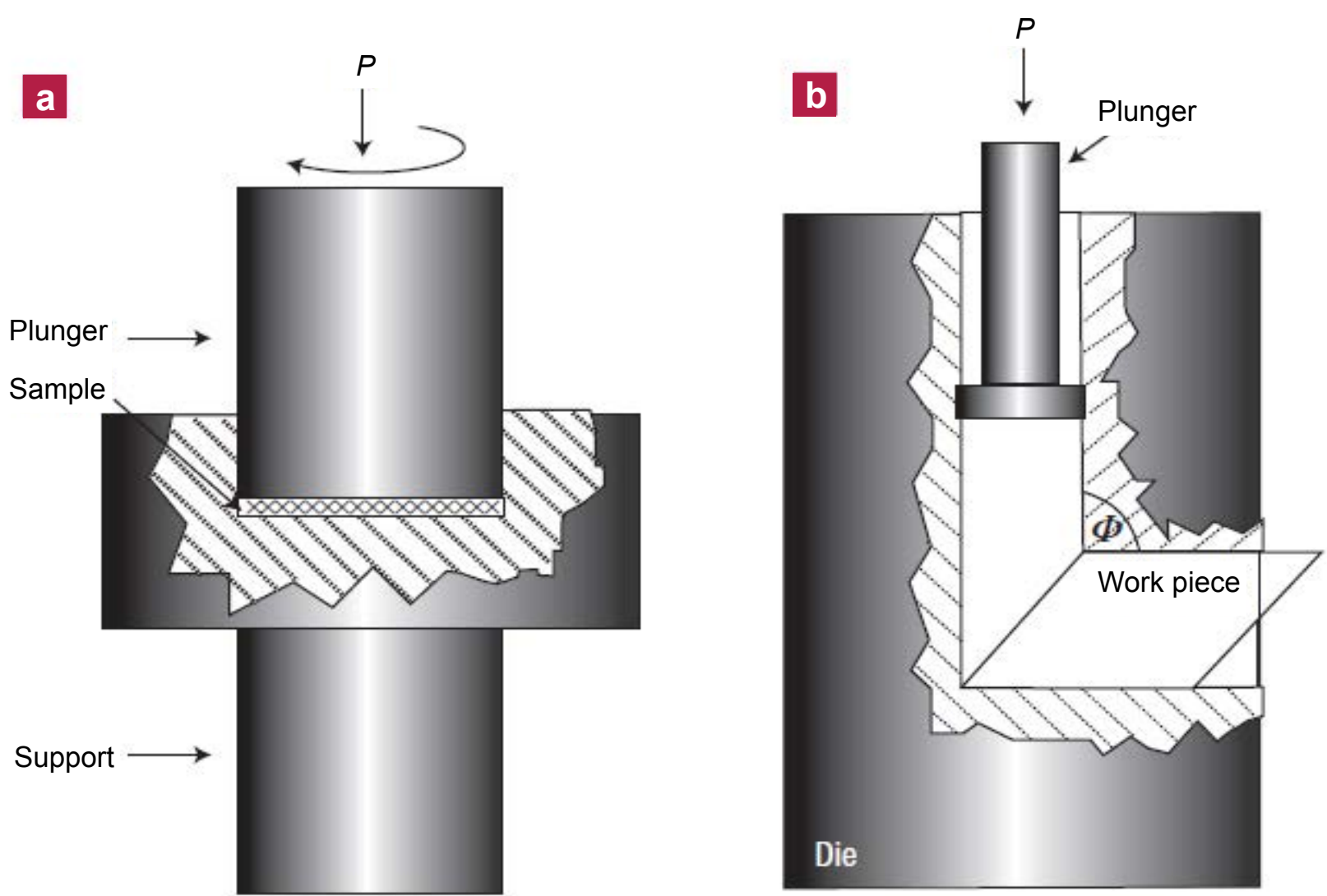

Figure 3: Two SPD techniques a) High-pressure torsion; b) Equal channel angular pressing (Adapted from [52]).

employed pulse plating with narrow pulse width and high peak current density to increase the deposition current density and the nucleation rate. They found that at different current densities, different surface morphologies, grain sizes, textures, and hardness were obtained. For example, when the current density ranges from 300 to $60 \mathrm{~A} \mathrm{dm}^{-2}$, the grain sizes is between 50 and $200 \mathrm{~nm}$. Ebrahimi, et al. [44] produced nano-grained nickel film by electrodeposition using a sulfamate-based electrolyte. They investigated the effect of $\mathrm{pH}$ of the electrolyte on the grain size. The results showed that grain size reached minimum around $\mathrm{pH}=$ 4.8. Saber, et al. [45] studies the effect of pulse peak current density on the grain size and surface morphology of zinc deposits with additives. It showed that increasing current density significantly changed the surface morphology and decreased the grain size. Also the texture of zinc deposits changed with increasing current density. Villain, et al. [47] quantitatively studies the grain growth of nanocrystalline silver deposits by measuring the electrode potential versus polycrystalline silver. A parabolic law was obtained for the grain growth. Cheung, et al. [48] showed that the amount of iron co-deposited with nickel can affect the grain size, texture of the nanocrystalline Ni-Fe alloy. Initially, hardness increased with decreasing grain size following HallPetch relationship. However, when the grain size reach a critical value $18 \mathrm{~nm}$, the hardness of the materials deviated from regular Hall-Petch relationship.
Severe Plastic Deformation (SPD) breaks down the coarse grain into nano-size grain. As an alternative to inert gas condensation and mechanical alloying which have inherent drawbacks such as porosity and contamination, severe plastic deformation has been seen as a new approach for fabricating bulk nanostructured materials [52]. SPD-produced nanomaterial can be fully dense and the large geometrical dimensions make it a perfect candidate for mechanical properties test. Though the grain refinement can be done by any means of introducing large plastic strains in metals, two methods for a material subjected to SPD have received widespread attention. They are High-Pressure Torsion (HPT) and Equal Channel Angular (ECA) pressing (Figure 3). The details about these two techniques can be referred in Valiev's paper [52] published on Nature or other review papers on SPD $[5,16,53]$. The grain boundary structure of SPD-processed materials is an important factor in determining new mechanical behavior. High fraction of high angle grain boundaries has been observed in ultrafine grained nickel in reference [54].

\section{Plastic Deformation Mechanism}

For conventional polycrystalline materials, the grain size dependence of strength and hardness is well established as Hall-Petch relationship, which indicates that the yield stress and hardness is inverse to the square root 
of grain size [55-58]. This strengthening at reduced grain sizes is attributed to the pile-up of dislocations at grain boundaries. However, when it comes to Nanocrystalline (nc) regime, the conventional Frank-Read dislocation sources ceases to control the deformation because the stress to bow out a dislocation approaches the theoretical shear strength $[55,57]$.

As long as the plasticity is primarily caused by conventional dislocation activity, the grain refinement strengthening effect will always exist. In conventional polycrystalline metals, the grain refinement during deformation often accompanies the formation of texture, which is accomplished by the dislocation glide on multiple active slip systems in grains [55]. However, texturing was not observed in heavily cold-rolled nanocrystalline Pd [40]. This clearly indicates that conventional dislocation deformation mechanism may not explain the strengthening of nc materials. Therefore, Markmann [40], suggested grain boundary sliding and grain rotation as concurring processes to control the deformation of nc materials. However, the validity of their explanation has to be further verified by experimental results. Budrovic Z, et al. [59] used in situ peak profile analysis to investigate plastic deformation of electrodeposited nc nickel and found that the peak broadening is reversible upon unloading, which is different than the irreversible broadening caused by normal dislocation mediated processes. Hemker [56], further stated that when materials become nc regime, the increasing volume fraction of grain boundary will serve as highly effective dislocation sinks and sources and traditional dislocation sources cease to operate. He also pointed out that grain boundary sliding and partial dislocation emission mechanism, suggested by molecular dynamics study [60], is in good agreement with the experimental observations of twinning and stacking fault in nc aluminum by Chen, et al. [61]. Therefore, in situ $\mathrm{x}$-ray peak profile analysis is proved to be an important tool to study atomic scale deformation processes in nc materials. Though the deformation mechanism for nc materials is still under debate, it is clear that the dislocation activity is fundamentally different in nc materials and a new mechanism should be responsible for the plastic deformation of nc materials.

$\mathrm{Ma}$ [55] pointed out that dislocations are nucleated out of grain boundaries sources and traverse the grain and disappear into the opposing grain boundaries, with little chance of being stored inside the grains. Also the emission of partial dislocation from a grain boundary becomes energetically favored, leaving behind debris such as deformation twins and stacking faults. Shan, et al. [62] has also proposed that grain boundary-mediated plasticity such as grain boundary sliding, grain rotation substitutes for conventional dislocation nucleation and motion as the dominant deformation mechanism when grain sizes are reduced below a certain value. Schiotz, et al. [63] used molecular dynamics simulations to simulate plastic deformation of nc copper. They attributed the maximum strength at a grain size of 10 to $15 \mathrm{~nm}$ to a transition in the microscopic deformation mechanism from dislocation controlled plasticity in coarse-grained materials to grain boundary sliding in the nc materials. Swygenhoven [58] believed that dislocation sources and pile up are hardly expected to exist in nc materials because of an increasing percentage of grain boundary atoms and a grain boundary accommodation mechanism should be responsible for the plastic deformation. All these observations [55-66] confirmed that the grain boundary played a very important role in the plastic deformation of nc materials. There are three ways to investigate the plastic deformation behavior of nc materials. They are molecular dynamics study, experimental observations, theoretical modeling.

\section{Molecular dynamics study}

Direct experimental visualization techniques are intrusive and almost impossible to get the intact grain boundary structure information. In situ Transmission electron microscopy requires samples to have a thickness comparable to the grain size and therefore alters the grain boundary structure $[58,66]$. It is still not clear that what extent will this structure change have an effect on mechanical properties of nc materials. However, molecular dynamics simulations provide an alternate way to investigate the deformation mechanism involving millions of atoms, representing a small part of three-dimensional bulk nc materials $[55,56,58,66]$.

The first attempt to use molecular dynamics to study the deformation of nc materials was made by Schiøtz, et al. [67]. They studied the deformation of a nanometer sized wire containing multiple grain boundaries. The migration of grain boundaries was observed. The correlated stress-strain curves from simulations clearly indicated the grain boundaries may be of great importance to the mechanical properties of nc materials. In ref [68], Schiøtz, et al. simulated of the deformation of nc copper, which show a softening with decreasing grain size in the smallest grain size regime. The plastic deformation is mainly carried by grain boundary sliding, with only a minor part being caused by normal intragranular dislocation activity. He further investigated the effects of varying temperature, strain rate and porosity on the mechanical behavior of nc copper [69]. He observed the maximum strength of nc copper at a grain size of 10 to $15 \mathrm{~nm}$ (Figure 4) [63]. He believed that the maximum is correlated by a shift in the microscopic deformation mechanism from dislocation-mediated plasticity in the coarse-grained material to grain boundary sliding in the nc region. Yamakov, et al. [65] further pointed out that this maximum strength strongly depends on the stacking fault energy, the elastic properties of the metal, and 
the magnitude of the applied stress. They also proposed a deformation-mechanism map (Figure 5) for the mechanical behavior of nanocrystalline fcc metals at low temperature. They also emphasized that the crossover in the deformation mechanism arises from the length-scale competition between the grain size and the dislocation splitting distance, which was verified by their previous studies [70]. In ref [71], Derlet, et al. explained that the

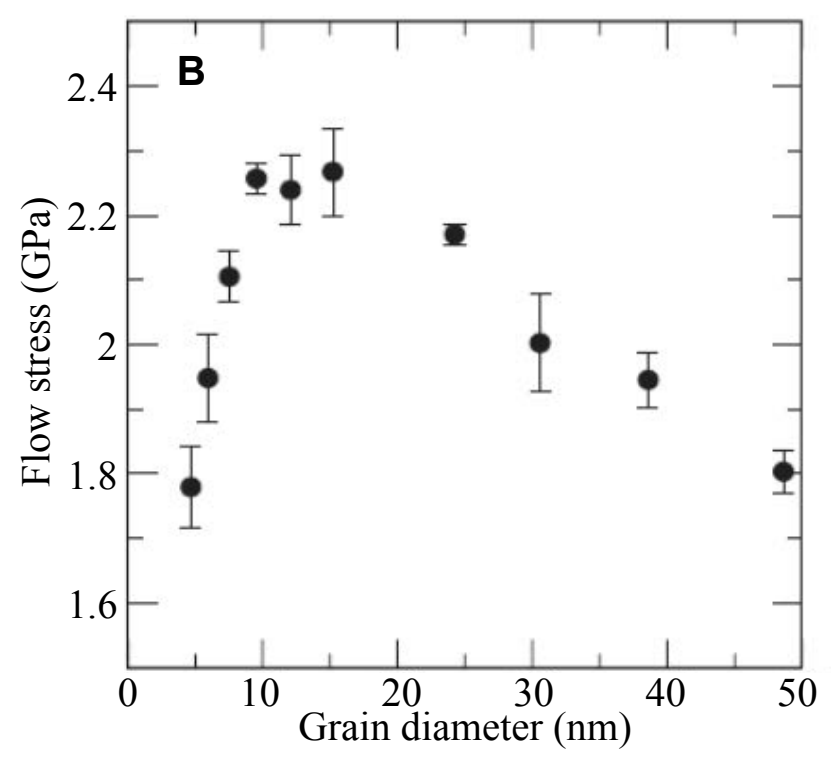

Figure 4: Molecular dynamics study of crossover regime of $\mathrm{nc} \mathrm{Cu}$. The diagram gives the grain size dependence of flow stress. The appearance of a maximum flow stress indicates a transition from dislocation mediated plastic deformation to grain boundary mediated plastic deformation. The decreased flow stress with decreasing grain size is known as inverse Hall-Petch relation (Adapted from Ref [63]). observed inverse grain size strain rate dependency in $\mathrm{Al}$ columnar structures in ref [70] is a result of the geometrical consequence in the nc regime grain. The dislocation activity difference in 2D and 3D can be explained by means of the geometrical restricted difference in available slip systems and the atomic activity observed in the grain boundary. In ref [72], Jacobsen, et al. analyzed the work done by Yamakov, et al. [70]. They pointed out that the interplay between grain boundaries and dislocations is critical to understand the mechanical properties of $\mathrm{nc}$ materials. A possibility to engineer the structure of grain boundaries to control the dislocation behavior and thereby the mechanical properties was proposed. In ref [73], Yip summarized the work done by Yamakov, et al. in [65] and provided more evidence for a crossover deformation mechanism. In ref [74], this crossover in deformation mechanism was observed to be accompanied by an obvious transition in the mechanical behavior of nc Al. Li, et al. [75] analyzed the completing mechanism between grain boundary and dislocation mediated deformation of nc Al during plastic strain recovery. The results showed that grain boundary played important role in the deformation of $\mathrm{nc} \mathrm{Al}$.

The map use the normalized stress and grain size, in which $\sigma_{\infty} \mathrm{r}_{0}$ are functions of SFE and elastic properties of the material. The two diagonal lines stand for 'dislocation splitting line' and 'nucleation stress line', defined by length scale competition between grain size and dislocation splitting distance and the grain size dependence of dislocation nucleation stress, respectively. The map couples the mechanical properties of nc fcc metals with the
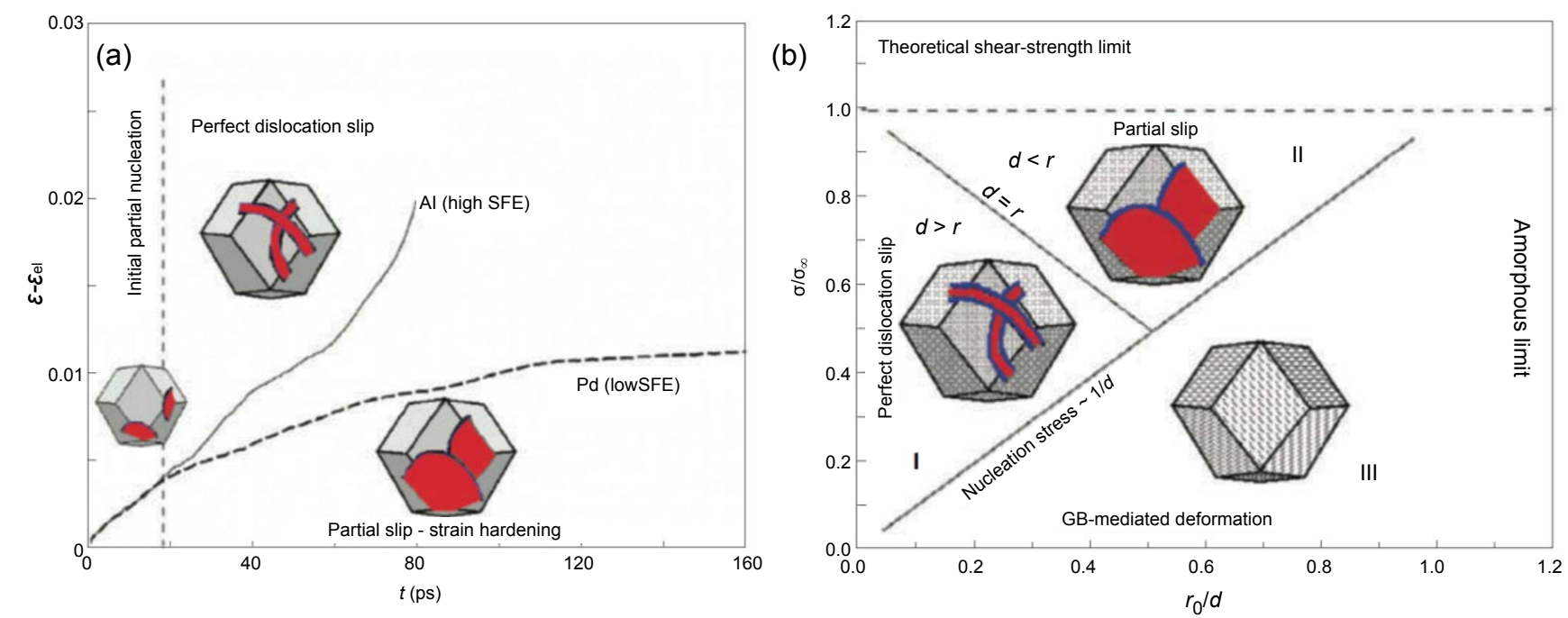

Figure 5: a) The comparison of time dependent plastic strains for a high SFE Al and low SFE Pd materials for a grain size of $24 \mathrm{~nm}$. After 1\% plastic strain, the strain rate in Al is almost constant while the strain rate in low SFE Pd decreases steadily. The curve begins to diverge at around $0.4 \%$ strain when the high SFE Al enter the perfect dislocation slip regime and in low SFE Pd, stacking faults continue to extend though gliding until the entire grain is transected. This inability to produce perfect dislocation in low SFE materials leads to strain hardening; b) The deformation-mechanism map proposed by Yamakov, et al. [65]. The map shows three distinct regions for low temperature deformation behavior of nc fcc metals. Region I, II, III is complete extended dislocations, partial dislocation, grain boundary dominated deformation, respectively. 
structure and physics of the dislocations (Adapted from Ref [65]).

In ref [76], the limitations of molecular dynamics method were brought forward by Swygenhoven, et al. They proposed a generalized stacking fault energy curve to interpret the nature of slip in nc metals. In ref [77], they simulated $\mathrm{nc} \mathrm{Ni}$ and addressed the microscopic mechanism of grain boundary sliding to be atomic shuffling and stress assisted free volume migration [56]. The accommodation processes under high stress and room temperature condition include grain boundary sliding, dislocation activity and triple junction migration. In refs $[60,78]$, Yamakov, et al. elucidated the interplay between dislocation and grain boundary processes during room temperature plastic deformation of nc Al. Deformation twins was observed, which was never shown in coarse-grain $\mathrm{Al}$ due to a high stacking fault energy. A new grain was formed near the triple junction, which can be explained by an intricate interplay between deformation twinning and dislocation nucleation from the grain boundaries during the deformation. They demonstrated that mechanical twinning may play an important role in the deformation behavior of nc Al. They also studied grain-boundary diffusion creep deformation mechanism in nc palladium [79]. The first observation of grain boundary sliding as an accommodation mechanism for coble creep was reported. They further analyzed the mechanisms of dislocation-dislocation and dislocation-twin boundary reactions during the deformation of $\mathrm{nc} \mathrm{Al}$ under sufficiently high stress [80]. These interactions may lead to complex twin networks, which may cause dislocation piles up and strain hardening. Jang, et al. [81] simulated the deformation of $\mathrm{nc}$ Al-Pb alloy. All the $\mathrm{Pb}$ was observed to be segregated at the grain boundary. The increasing $\mathrm{Pb}$ content impeded the nucleation of partial dislocation. The preferred grain boundary accommodation mechanism led to a decreased yield stress, which is contradictory with the normal solute hardening in coarse-grained materials. A detailed review about molecular dynamic studies on deformation mechanism of $\mathrm{nc}$ materials is given in ref [82].

Other atomistic simulation methods have also been used to study the deformation mechanism of nc materials. Tadmor, et al. [83] used the quasicontinuum method to simulate large-scale atomic resolution of nanoindentation into a thin aluminum film to study the initial stages of plastic deformation. They observed two different deformation mechanisms, nucleation and movement of edge dislocation dipoles and deformation twinning. Farkas, et al. [84] applied molecular statics with Embedded Atom Method interatomic potentials to study the dislocation emission process and the grain boundary sliding process and their effects on the stress-strain curves in nc $\mathrm{Ni}$. Both dislocation emission from the grain boundaries and grain boundary sliding was observed.
The limitations of molecular dynamics study were described by $[55,56,58,66,71,76,79,82]$. The simulations were performed under high stresses, low temperature and very short deformation time [55-58,71]. The time dependent processes like thermal activated process, which may contribute to the deformation in real world experiments, were excluded from simulations $[55,57,58,76]$. In ref [79], Yamakov, et al. tried to perform the simulation at elevated temperature. The observed grain boundary sliding via coble diffusion creep seems to verify the existence of rate limiting processes. However, for room temperature simulations, the contribution from these rate limiting processes is still unknown. Therefore, the deformation mechanism at low temperature is still unclear by molecular dynamics studies. Ref [66] pointed out that the essentially two dimensional nature of the simulations by Yamakov, et al. $[60,70,78-80]$ could affect the final results because of the reduced number of possible slip systems. Also, ref [57] pointed out that most simulations have been performed on pure samples containing defect free grains with high-angle grain boundaries and a narrow grain size distribution. This neglects the influence of impurities and low-angle grain boundaries or other types of special boundaries. Molecular dynamics studies can only be taken as an inspiration or guidance, but could not directly validate the existence of a mechanism. In ref [85], Mohamed, et al. proposed five requirements for a successful deformation mechanism based on the available experimental data.

\section{Experimental verifications}

The inability of molecular dynamics simulations to accurately verify the deformation mechanisms makes the experimental observation irreplaceable to unlock the secrets of plastic deformation of nc materials [55]. Two experimental observation methods, in situ TEM [86] and in situ $\mathrm{X}$-ray peak profile analysis $[59,87]$ were used to investigate the unusual deformation behavior of nc materials. Chen, et al. [61] first used the TEM to provide direct evidence of the presence of deformation twinning predicted by Yamakov, et al. [78] in molecular dynamics simulation in plastically deformed $\mathrm{nc}$ Al. Shan, et al. [62] used in situ dynamic TEM to study nc Ni films with an average $10 \mathrm{~nm}$ grain size. The grain boundary mediated deformation is believed to be the dominant deformation mode. The extremely short lifetime of emitted and reabsorbed dislocations predicted by molecular dynamics explains why they are hard to be observed by TEM [56]. The in situ $\mathrm{x}$-ray peak profile analysis seems to compensate the inability of in situ TEM to capture the transient dynamic processes [59]. The suggested mechanism-atomic shuffling or free volume migration in the grain boundaries, which is responsible for grain boundary sliding and partial dislocation emission, is possible to be detected by peak broadening measurements [59] 
because they changed the structure of grain boundaries [56]. Ref [55] also proposed that the trailing partial dislocation emission can erase the stacking fault left by the leading partial dislocation and therefore combined into full dislocation, which is hard to observe by TEM since they leave no footprint after transecting the grain. The sharpened diffraction peaks in ref [59] are a good indication of structural rearrangement of grain boundaries during plastic deformation. The unusual reversible peak broadening phenomenon in $\mathrm{nc} \mathrm{Ni}$ broadening demonstrated the lack of a permanent residual dislocation network. This seems to imply an undiscovered deformation mechanism other than conventional dislocation motion.

Kumar, et al. [86] investigated the mechanisms of deformation and damage in electrodeposited, fully dense, $\mathrm{nc} \mathrm{Ni}$ with an average grain size of $30 \mathrm{~nm}$ and a narrow grain size distribution by in situ and ex situ TEM observations. The observations clearly showed that dislocation-mediated deformation played a dominant role in the deformation of nc Ni. Wang, et al. [88] used postmortem transmission electron microscopy to study the deformation mechanism of $\mathrm{nc} \mathrm{Ni}$ with grain size distribution from 30 to $100 \mathrm{~nm}$ under very high strain rates. No deformation twinning was observed. The results revealed that dislocation activity was a primary deformation mechanism for the studied nc Ni. Jang, et al. [89] studied the plastic deformation of nc Fe. For grain sizes above about $18 \mathrm{~nm}$, the Hall-Petch relation was observed and slight softening began with further decreasing grain sizes. The strain rate sensitivity increases with decreasing grain size. Chen, et al. [90] measured the hardness of $\mathrm{nc} \mathrm{Cu}$ and found that the Hall-Petch relation was obeyed until grain size as small as $10 \mathrm{~nm}$. The further investigation of strain rate sensitivity and a flow stress activation volume indicated that grain boundary activity was enhanced at grain size $10 \mathrm{~nm}$ but not a primary deformation mechanism. Cross, et al. [91] got direct experimental data from nanoindentation tests and their results suggested a new mechanism at reduced grain size, which suppressed permanent mass transport. Zied, et al. [92] investigated the effects of pressure on microstructure and mechanical properties of compacted ball-milled aluminum. The results showed that the dislocation density and micro-hardness of our specimens increased with increasing pressure. Li, et al. [93] used in situ synchrotron diffraction technique to study nanostructured $\mathrm{Ni}-\mathrm{Fe}$ alloy. They found that plastic deformation went through a transition with applied strain. The deformation was mainly accommodated by grain boundaries at low strains, while at large strains, the dislocation motion became active and dominated. They also pointed out that $0.2 \%$ offset criterion was not applicable to define the macroscopic yield strength at small nc grain size.

\section{Twinning}

Deformation twinning, as an important deformation mechanism in nc materials, can significantly affect their

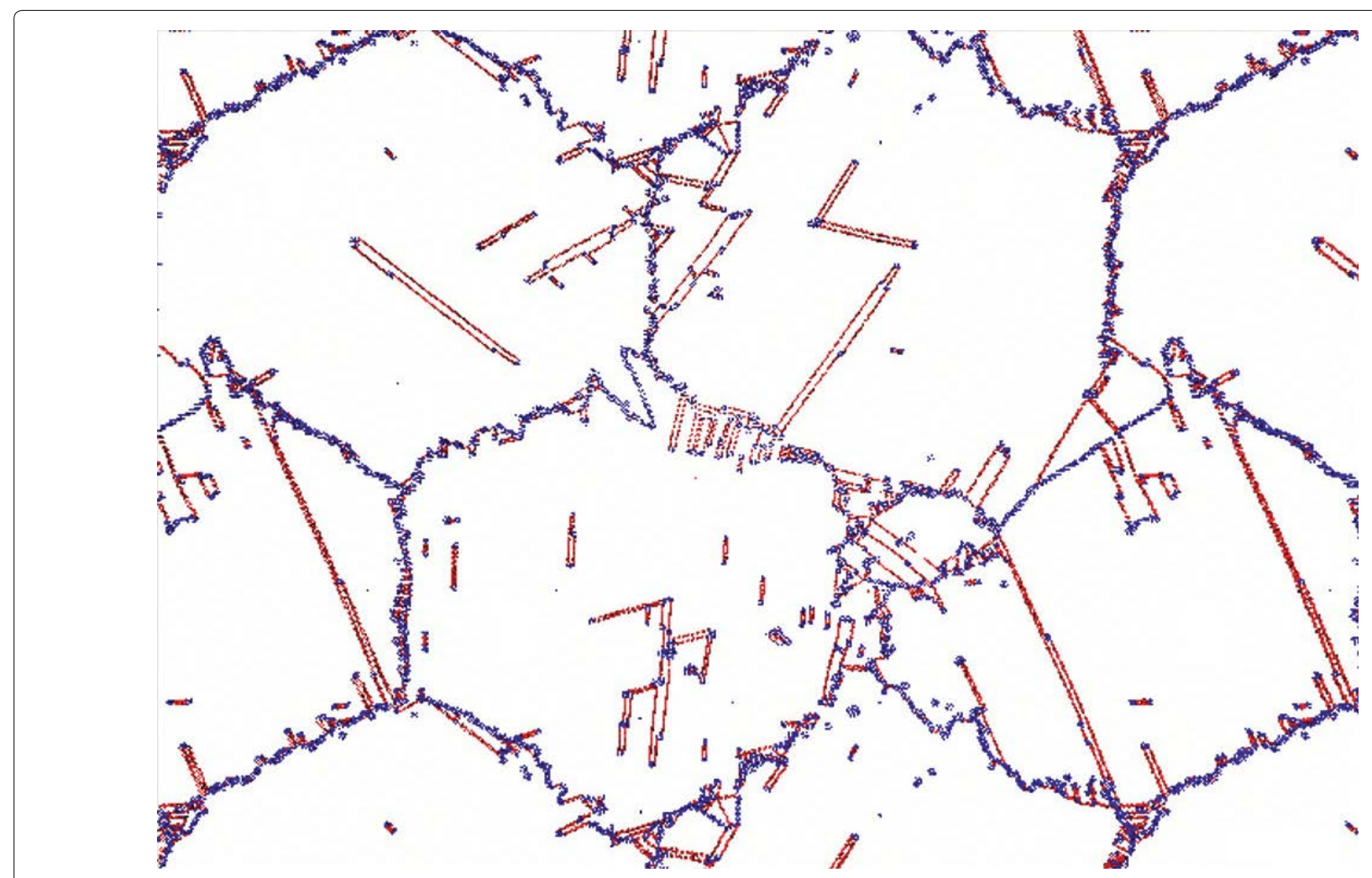

Figure 6: Deformation twinning revealed in MD simulation snapshot of nc Al by Yamakov, et al. [78]. Various interactions between dislocations and grain boundaries have been observed. Twins are formed by successive emission of Shockley partials from grain boundary. The triple junction shows a formation of a new grain (Adapted from Ref [78]). 
mechanical properties [94]. The deformation twinning in coarse grained fcc metals are believed to be done by pole mechanism, prismatic glide mechanism, faulted dipole mechanism, which require an intra-grain dislocation source to operate [95]. However, as grain size enters into nc regime, the resolved shear stress to bow out a FrankRead dislocation is approaching theoretical shear stress. Therefore, intra-grain dislocation source is hard to operate and multiply. Few dislocations were observed in nc grains [94]. In coarse grained fcc metals, the stress for twinning increases faster than the stress for perfect dislocation slip. Smaller grain size makes the deformation twinning more difficult [96]. Lower temperature and higher strain rate usually promote deformation twinning [95,96]. A detailed review about deformation twinning in coarse grained metals is provided in ref [95]. However, the formation of deformation twins in nc metals shows a different picture than that in coarse grained metals.

Deformation twins were first predicted via quasicontinuum method by Tadmor, et al. [83]. Later, Yamakov, et al. $[60,78,80]$ used massively parallel molecular dynamics and observed the presence of deformation twins in $\mathrm{nc}$ $\mathrm{Al}$ during the intricate interplay between dislocation and grain boundary mediated deformation processes (Figure 6). Chen, et al. [61] first used TEM to observe directly the existence of deformation twins in $\mathrm{nc} \mathrm{Al}$ (Figure 7).

The twinning mechanisms in nc metals are proposed to be primarily partial dislocation emission from grain boundary [94]. Wu, et al. [97], Feng, et al. [98] confirmed that twinning does occur upon large plastic deformation in $\mathrm{nc} \mathrm{Ni}$. They both provided compelling evidence that grain boundary emission of partial dislocation is the dominating twinning mechanism for $\mathrm{nc} \mathrm{Ni}$. This is consistent with molecular dynamics results of $\mathrm{nc}$ fcc metals by Froseth, et al. [99], Wu, et al. also observed other twinning mechanisms like dynamic overlapping of extended partial dislocations and grain boundary splitting and migration. This further confirmed the observations of Rosner, et al. [100] that grain boundary sliding and grain rotation are actively involved with twinning. Liao, et al. [101] used high resolution TEM to confirm that twins and stacking faults in $\mathrm{nc} \mathrm{Cu}$ were formed through partial dislocation emissions from grain boundaries, which is different from the pole mechanism in coarse-grained copper. Wang, et al. [102] investigated the deformation mechanism of nc bcc Ta and found that the deformation process is controlled by twinning. They also pointed out that the physical mechanism of deformation twinning in $\mathrm{nc}$ bcc metals is different than that in nc fcc metals. More details can be obtained in ref [94].

Detwinning has been observed both experimentally and in molecular-dynamics simulations, which will significantly soften the material and may lead to accelerated failure [103]. Grain size can have a significant effect
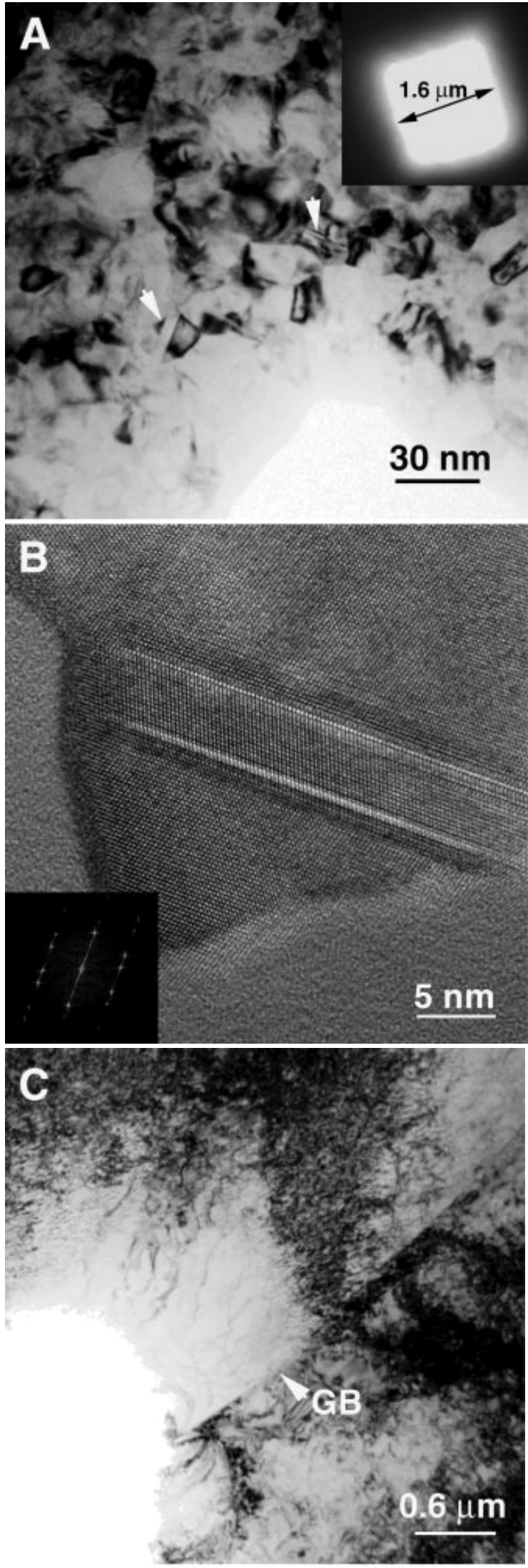

Figure 7: A) Deformation twins around an indent in nc Al observed by TEM; B) HRTEM micrograph showing a deformation twin in $(A)$ with parallel twin boundaries which are several atomic planes thick; C) Many dislocations around grain boundary were observed in coarse-grained pure aluminum. No evidence of deformation twins (Adapted from Ref [61]). 
on the competition between twinning and detwinning [103] Twinning propensity dominated in a certain range of grain sizes, while the detwinning process dominated outside of this range to annihilate all twins [103]. Nc fcc metals have been reported to deform by twinning when their grains are smaller than a certain size. However, when their grain sizes were very small, an inverse grainsize effect, i.e. twinning becomes more difficult with decreasing grain size, was observed [103-105].

The tendency for detwinning is stronger at small grain sizes (e.g. below $35 \mathrm{~nm}$ ) than that at large grain sizes (e.g. larger than $100 \mathrm{~nm}$ ), due to the relatively high energy barriers needed for detwinning to overcome dislocation interactions with twin boundaries. The detwinning process is caused primarily by the dislocation interactions with twins so that it is more readily activated in smaller grains due to their higher flow stresses [103]. Understanding the effect of grain size on deformation twinning and detwinning would allow us to predict the stability and evolution of microstructures and design nanocrystalline twin materials that enhance the strength and ductility $[103,104,106]$.

Analytical expressions were obtained for the grain size corresponding to the peak of the twinning propensity, the required external stress for twinning propagation in polycrystals and the grain size at which the slip begins to prevail over the twinning $[107,108]$. The grain size range of the nanotwinning propensity, the grain size of its peak, and the external twinning stress are calculated for nanocrystalline metals with different crystal structures such as fcc, bcc, hcp [107]. The theoretical results were compared with the experiments for twinning in $\mathrm{Ni}$ and $\mathrm{Cu}$ [108].

\section{Other Properties of Nanocrystalline Materials}

It might be interesting to briefly mention other grain size dependent properties of nanocrystalline materials. The effect of grain size on the elastic modulus of nanocrystalline materials has been studied by Sharma, et al. [109]. They proposed a closed form model to calculate the grain size dependent elastic modulus of nc materials by considering grain boundary sliding. A mechanics model of nanocrystalline nanowires has been proposed by Shaat, et al. [110] to calculate the ranges of the elastic properties and buckling strength of diamond, $\mathrm{Si}, \mathrm{Al}$, $\mathrm{Cu}, \mathrm{Ag}, \mathrm{Au}$, and $\mathrm{Pt}$ nanowires. They calculated the range of Young's modulus, shear modulus, bulk modulus, and mass density of these nanowires for each grain size (from $200 \mathrm{~nm}$ to $2 \mathrm{~nm}$ ). They also reported the size dependent properties of nanowires due to their surface effect by calculating the equivalent Young's modulus and buckling strength of nanowires with different sizes, which are experimentally validated.

The effect of grain size on the electrical resistivity/ conductivity in nanoscale polycrystalline metallic materials has been studied by Tian, et al. $[111,112]$. The electrical conductivity of polycrystalline materials decreased obviously when the grain size is comparable with the electron mean free path inside the grain interior due to the significant contribution of grain boundary scattering to resistivity [113].

\section{Conclusions}

The unusual mechanical properties of nanocrystalline materials are strongly related to the grain size dependent nano-scale structure. The high volume fraction of grain boundary phase enables grain boundary to play a dominating role in the plastic deformation of nc materials. In contrast, conventional plastic deformation from intra-grain dislocation activity is strongly restricted because the resolved shear stress to bow out a dislocation source in the grain is approaching theoretical shear stress due to the small grain size. However, in the nanocrystalline regime, deformation mechanism of partial dislocation emission from grain boundaries resulting in strain hardening compete with grain boundary accommodation mechanism, which is responsible for stress softening, known as inverse HallPetch relation. This competition is reflected by the length scale competition between grain size and dislocation splitting distance. The competition leads to a crossover in deformation mechanism as well as mechanical properties of nc materials, which depends on the grain size, stacking fault energy, applied stress and elastic properties of materials. It is also important to realize that the grain size distribution and flaws introduced by different fabrication techniques can also exert some effects on the mechanical properties of nc material.

\section{References}

1. R Birringer, U Herr, H Gleiter (1986) Nanocrystalline Materials-a First Report. Trans Jpn Inst Met 27: 43-52.

2. Gleiter H (2000) Nanostructured materials: Basic concepts and microstructure. Acta Materialia 48: 1-29.

3. R Birringer (1989) Nanocrystalline materials. Materials Science and Engineering 117: 33-43.

4. SK Kumar, HV Swygenhoven, S Suresh (2003) Mechanical behavior of nanocrystalline metals and alloys. Acta Materialia 51: 5743-5774.

5. MA Meyers, A Mishra, DJ Benson (2006) Mechanical properties of nanocrystalline materials. Progress in Materials Science 51: 427-556.

6. H Gleiter (1991) Nanocrystalline solid. J Appl Cryst 24: 79-90.

7. C Suryanarayana (1995) Nanocrystalline materials. International Materials Reviews 40: 41-64.

8. H Gleiter (1991) Nanocrystalline Material. Advanced Structural and Functional Materials 1-37.

9. H Gleiter (1992) Materials with ultrafine microstructures: retrospective and perspectives. Nanostructured Materials 1: 1-19. 
10. JR Weertman, D Farkas, K Hemker, H Kung, M Mayo, et al. (1999) Structure and mechanical behavior of bulk nanocrystalline materials. MRS Bulletin 24: 44-53.

11. CC Koch (1993) The synthesis and structure of nanocrystalline materials produced by mechanical attrition: A review. Nanostructured Materials 2: 109-129.

12. C Suryanarayana, FH Froes (1992) The structure and mechanical properties of metallicnanocrystals. Metallurgical and Materials Transactions A 23: 1071-1081.

13. C Suryanarayana (1994) Nanocrystalline materials - A critical review, High temperature high performance materials for rocket engines and space applications. Proceedings of the Symposium 61-119.

14. K Lu (1996) Nanocrystalline metals crystallized from amorphous solids: nanocrystallization, structure, and properties. Materials Science and Engineering: R: Reports 16: 161-221.

15. C Suryanarayana, CC Koch (2000) Nanocrystalline materials - Current research and future directions. Hyperfine Interactions 130: 5-44.

16. RZ Valiev, RK Islamgaliev, IV Alexandrov (2000) Bulk nanostructured materials from severeplastic deformation. Progress in Materials Science 45: 103-189.

17. Mayo MJ (1993) Synthesis and applications of nanocrystalline ceramics. Materials \& Design 14: 323-329.

18. MJ Mayo (1996) Processing of nanocrystalline ceramics from ultrafine particles. International Materials Reviews 41: 85-115.

19. MJ Mayo, DC Hague, DJ Chen (1993) Processing nanocrystalline ceramics for applications in superplasticity. Materials Science and Engineering A 166: 145-159.

20. MJ Mayo (1997) High and Low temperature superplasticity in nanocrystalline materials. Nanostructured Materials 9: 717-726.

21. AV Sergueeva, C Song, RZ Valiev, AK Mukherjee (2003) Structure and properties of amorphous and nanocrystalline NiTiprepared by severe plastic deformation and annealing. Materials Science and Engineering A 339: 159-165.

22. RZ Valiev, AV Sergueeva, AK Mukherjee (2003) The effect of annealing on tensile deformation behaviorof nanostructured SPD titanium. Scripta Materialia 49: 669-674.

23. JE Carsley, A Fisher, WW Milligan, EC Aifantis (1998) Mechanical Behavior of a Bulk Nanostructured Iron Alloy. Metallurgical and Materials Transactions A 29: 2261-2271.

24. Khaled M Youssef, Ronald O Scattergood, K Linga Murty (2005) Ultrahigh strength and high ductility of bulk nanocrystalline copper. Applied Physics Letters 87.

25. DS Lashmore, WA Jesser, DM Schladitz, HJ Schladitz, HGF Wilsdorf (1977) Microstructural investigation of polycrystalline iron whiskers. J Appl Phys 48.

26. GW Nieman, JR Weertman, RW Siegel (1991) Mechanical behavior of nanocrystalline $\mathrm{Cu}$ and $\mathrm{Pd}$. J Mater Res 6: 1012-1027.

27. AV Sergueeva, VV Stolyarov, RZ Valiev, AK Mukherjee (2001) Advanced mechanical properties of pure titanium withultrafine grained structure. Scripta Materialia 45: 747752.

28. GE Fougere, JR Weertman, RW Siegel (1995) Processing and mechanical behavior of Nanocrystalline Fe. Nanostructured Materials 5: 127-134.
29. SR Agnew, BR Elliott, CJ Youngdahl, KJ Hemker, JR Weertman (2000) Microstructure and mechanical behavior of nanocrystalline metals. Materials Science and Engineering A 285: 391-396.

30. BQ Han, J Ye, F Tang, J Schoenung, EJ Lavernia (2007) Processing and behavior of nanostructured metallic alloys andcomposites by cryomilling. J Mater Sci 42: 1660-1672.

31. KW Liu, F Mücklich (2001) Thermal stabilityof nano-RuAl produced by mechanical alloying. Acta Materialia 49: 395403.

32. Mahmoud Zawrah, Leon Shaw (2003) Microstructure and hardness of nanostructured Al-Fe-Cr-Ti alloys through mechanical alloying. Materials Science and Engineering: A 355: $37-49$

33. Young S Parka, Kyung H Chunga, Nack J Kimb, Enrique J Lavernia (2004) Microstructural investigation of nanocrystalline bulk Al-Mg alloy fabricated by cryomilling and extrusion. Materials Science and Engineering: A 374: 211-216.

34. Yoritoshi Minamino, Yuichiro Koizumi, Nobuhiro Tsuji, Naoko Hirohata, Kiyoshi Mizuuchi, et al. (2004) Microstructures and mechanical properties of bulk nanocrystalline Fe-Al-C alloys made by mechanically alloying with subsequent spark plasma sintering. Science and Technology of Advanced Materials 5: 133-143.

35. GW Nieman, JR Weertman, RW Siegel (1989) Micro-hardness of nanocrystalline palladium and copper produced by inert-gas condensation. Scripta Metallurgica 23: 2013-2018.

36. H Kisker, T Gessmann, R Wurschum, H Kronmüller, HE Schaefer (1995) Magnetic properties of high purity nanocrystalline nickel. Nanostructured Materials 6: 925-928.

37. H Chang, CJ Altstettera, RS Averback (1992) Characteristics of nanophase TiAl produced by inert gas condensation. Journal of Materials Research 7: 2962-2970.

38. J Mühlbach, E Recknagel, K Sattler (1981) Inert gas condensation of $\mathrm{Sb}, \mathrm{Bi}$ and $\mathrm{Pb}$ clusters. Surface Science 106: 188-194.

39. G Sanders, GE Fougere, LJ Thompson, JA Eastman, JR Weertman (1997) Improvements in the Synthesis and Compaction of Nanocrystalline Material. Nanostructured Materials 8: 243-252.

40. J Markmann, P Bunzel, H Rösner, KW Liu, KA Padmanabhan, et al. (2003) Microstructure evolution during rolling of inert-gas condensed palladium. Scripta Materialia 49: $637-644$

41. Frank Endres, Mirko Bukowski, Rolf Hempelmann, Harald Natter (2003) Electrodeposition of Nanocrystalline Metals and Alloys from Ionic Liquids. Angewandte Chemie International Edition 42: 3428-3430.

42. Ning Wang, Zhirui Wang, KT Aust, U Erb (1997) Room temperature creep behavior of nanocrystalline nickel produced by an electrodeposition technique. Materials Science and Engineering: A 237: 150-158.

43. NS Qu, D Zhu, KC Chan, WN Lei (2003) Pulse electrodeposition of nanocrystalline nickel using ultra narrow pulse width and high peak current density. Surface and Coatings Technology 168: 123-128

44. F Ebrahimi, GR Bourne, MS Kelly, TE Matthews (1999) Mechanical properties of nanocrystalline nickel produced by electrodeposition. Nanostructured Materials 11: 343-350. 
45. Kh Saber, CC Koch, PS Fedkiw (2003) Pulse current electrodeposition of nanocrystalline zinc. Materials Science and Engineering: A 341: 174-181.

46. H Natter, R Hempelmann (1996) Nanocrystalline Copper by Pulsed Electrodeposition: The Effects of Organic Additives, Bath Temperature, and $\mathrm{pH}$. J Phys Chem 100: 19525-19532.

47. S Villain, P Knauth, G Schwitzgebel (1997) Electrodeposition of nanocrystalline silver: Study of grain growth by measurement of reversible electromotive force. J Phys Chem B 101: 7452-7454.

48. C Cheung, F Djuanda, U Erb, G Palumbo (1995) Electrodeposition of nanocrystalline Ni-Fe alloys. Nanostructured Materials 5: 513-523.

49. T Yamasaki (2001) High-strength nanocrystalline Ni-W alloys produced by electrodeposition and their embrittlement behaviors during grain growth. Scripta Materialia 44: 1497-1502.

50. I Baskarana, Sankara Narayanan, A Stephen (2006) Pulsed electrodeposition of nanocrystalline $\mathrm{Cu}-\mathrm{Ni}$ alloy films and evaluation of their characteristic properties. Materials Letters 60: 1990-1995.

51. Hajime Iwasaki, Kenji Higashi, TG Nieh (2004) Tensile deformation and microstructure of a nanocrystalline $\mathrm{Ni}-\mathrm{W}$ alloy produced by electrodeposition. Scripta Materialia 50: 395-399.

52. Valiev R (2004) Nanostructuring of metals by severe plastic deformation for advanced properties. Nature Materials 3: 511-516.

53. RZ Valiev, AV Korznikov, RR Mulyukov (1993) Structure and properties of ultrafine-grained materials produced bysevere plastic deformation. Materials Science and Engineering A 168: 141-148.

54. AP Zhilyaev, BK Kim, GV Nurislamova, MD Bar, JA Szpunar, et al. (2002) Orientation imaging microscopy of ultrafine-grained nickel. Scripta Materialia 46: 575-580.

55. Ma E (2004) Watching the Nanograins Roll. Science 305: 623-624.

56. KJ Hemker (2004) Understanding How Nanocrystalline Metals Deform. Science 304: 221-223.

57. HV Swygenhoven, Weertman JR (2006) Deformation in nanocrystalline metals. Materialstoday 9: 24-31.

58. HV Swygenhoven (2002) Grain Boundaries and dislocations. Science 296: 66-67.

59. Z Budrovic, HV Swygenhoven, PM Derlet, SV Petegem, B Schmitt (2004) Plastic Deformation with Reversible Peak Broadening in Nanocrystalline Nickel. Science 304: 273276.

60. V Yamakov, D Wolf, SR Phillpot, H Gleiter (2002) Deformation twinning in nanocrystalline Al by molecular-dynamics simulation. Acta Materialia 50: 5005-5020.

61. MW Chen, Ma E, KJ Hemker, HW Sheng, YM Wang, et al. (2003) Deformation Twinning inNanocrystalline Aluminum. Science 300: 1275-1277.

62. ZW Shan, EA Stach, JMK Wiezorek, JA Knapp, DM Follstaedt, et al. (2004) Grain Boundary-Mediated Plasticity in Nanocrystalline Nickel. Science 305: 654-657.

63. J Schiøtz, KW Jacobsen (2003) A Maximum in the Strength of Nanocrystalline Copper. Science 301: 1357-1359.
64. MY Gutkin, IA Ovid'ko, CS Pande (2001) Theoretical models of plastic deformation processes in nanocrystalline materials. Rev Adv Mater Sci 2: 80-102.

65. V Yamakov, D Wolf, SR Phillpot, AK Mukherjee, H Gleiter (2004) Deformation-mechanism map for nanocrystalline metals by molecular-dynamics simulation. Nature Materials 3: 4347.

66. Bilde-Sørensen, J Schiotz (2003) Nanocrystals Get Twins. Science 300: 1244-1245.

67. J Schiøtz, T Rasmussen, KW Jacobsen, OH Nielsen (1996) Mechanical deformation of nanocrystalline materials. Phil Mag Lett 74: 339-344.

68. Jakob Schiøtz, Francesco D Di Tolla, Karsten W Jacobsen (1998) Softening of nanocrystalline metals at very small grain sizes. Nature 391: 561-563.

69. J Schiøtz, T Vegge, FD Di Tolla, KW Jacobsen (1999) Atomic-scale simulations of the mechanical deformation of nanocrystalline metals. Phys Rev B 60: 11971-11983.

70. V Yamakov, D Wolf, M Salazar, SR Phillpot, H Gleiter (2001) Length-Scale Effects in the Nucleation of Extended Dislocations in Nanocrystalline Al by Molecular-Dynamics Simulation. Acta Materialia 49: 2713-2722.

71. PM Derlet, H Van Swygenhoven (2002) Length scale effects in the simulation of deformation properties of nanocrystalline metals. Scripta Materialia 47: 719-724.

72. W Karsten Jacobsen, Jakob Schi (2002) Nanoscale plasticity. Nature Materials 1: 15-16.

73. Sidney Yip (2004) Mapping plasticity. Nature Materials 3: 11-12.

74. V Yamakov, D Wolf, SR Phillpot, AK Mukherjee, H Gleiter (2003) Deformation mechanism cross over and mechanical behaviour in nanocrystalline materials. Philosophical Magazine Letters 83: 385-393.

75. Xiaoyan Lia, Yujie Weia, Wei Yang, Huajian Gao (2009) Competing grain-boundary- and dislocation-mediated mechanisms in plastic strain recovery in nanocrystalline aluminum. Proc Natl Acad Sci 106: 16108-16113.

76. H Van Swygenhoven, PM Derlet, AG Frøseth (2004) Stacking fault energies and slip in nanocrystalline metals. Nat Mater 3: 399-403.

77. H Van Swygenhoven, PM Derlet (2001) Grain-boundary sliding in nanocrystalline fcc metals. Physics Review B 64: 224105.

78. Vesselin Yamakov, Dieter Wolf, Simon R Phillpot, Amiya K Mukherjee, Herbert Gleiter (2002) Dislocation processes in the deformation of nanocrystalline aluminium by molecular-dynamics simulation. Nature Materials 1: 45-49.

79. V Yamakov, D Wolf, M Salazar, SR Phillpot, H Gleiter (2001) Grain Boundary Diffusion Creep in Nanocrystalline Palladium by Molecular-Dynamics Simulation. Acta Materialia 50: 61-73.

80. V Yamakov, D Wolf, SR Phillpot, H Gleiter (2003) Dislocation-dislocation and dislocation-twin reactions in nanocrystalline Al by molecular dynamics simulation. Acta Materialia 51: 4135-4147.

81. S Jang, Y Purohit, D Irving, C Padgett, D Brenner, et al. (2008) Molecular dynamics simulations of deformation in nanocrystalline $\mathrm{Al}-\mathrm{Pb}$ alloys. Materials Science and Engineering A 493: 53-57. 
82. D Wol, V Yamakov, SR Phillpot, A Mukherjee, H Gleiter (2005) Deformation of nanocrystalline materials by molecular-dynamicssimulation: relationship to experiments. Acta Materialia 53: 1-40.

83. EB Tadmor, R Miller, R Phillips (1999) Nanoindentation and incipient plasticity. Journal of Materials Research 14: 22332250.

84. D Farkas, WA Curtin (2005) Plastic deformation mechanisms in nanocrystalline columnar grain structures. Materials Science and Engineering A 412: 316-322.

85. A Farghalli Mohamed, Heather Yang (2010) Deformation Mechanisms in Nanocrystalline Materials. Metallurgical and Materials Transactions A 41: 823-837.

86. KS Kumar, S Suresh, MF Chisholm, JA Horton, P Wang (2003) Deformation of electro deposited nanocrystalline nickel. Acta Materialia 51: 387-405.

87. Tamas Ungar, Jeno Gubicza (2007) Nanocrystalline materials studied by powder diffraction line profile analysis. Z Kristallogr 222: 114-128.

88. YM Wang, EM Bring, JM McNaney, M Victoria, A Caro, et al. (2006) Deforming nanocrystalline nickel at ultrahigh strain rates. Applied Physics Letters 88: 061917.

89. D Jang, M Atzmon (2003) Grain-size dependence of plastic deformation in nanocrystalline Fe. Journal of Applied Physics 93: 9282-9286.

90. J Chen, L Lu, K Lu (2006) Hardness and strain rate sensitivity of nanocrystalline Cu. Scripta Materialia 54: 1913-1918.

91. Graham LW Cross, Andre Schirmeisen, Peter Grutter, Urst Durig (2006) Plasticity, healing and shakedown in sharp-asperity nanoindentation. Nat Mater 5: 370-376.

92. M Abou Zied, AA Ebnalwaled (2008) Microstructure and hardness dependence of the pressure of nano-crystalline aluminum. Intermetallics 16: 745-750.

93. Hongqi Li, Hahn Choo, Yang Ren, Tarik A Saleh, Ulrich Lienert, et al. (2008) Strain-Dependent Deformation Behavior in Nanocrystalline Metals. Physical Review Letters 101: 015502.

94. YT Zhu, XZ Liao, XL Wu (2012) Deformation twinning in nanocrystalline materials. Progress in Materials Science 57: 1-62.

95. JW Christian, S Mahajan (1995) Deformation twinning. Prog Mater Sci 39: 1-157.

96. MA Meyers, O Vohringer, VA Lubarda (2001) The onset of twinning in metals: a constitutive description. Acta Materialia 49: 4025-4039.

97. Xiao Lei Wu, En (Evan) Ma (2006) Deformation twinning mechanisms in nanocrystalline Ni. Applied Physics Letters 88: 061905.

98. XY Feng, ZY Cheng, X Wu, TC Wang, YS Hong (2006) Twinning in nanocrystalline $\mathrm{Ni}$ by severe plastic deformation. J Phys D: Appl Phys 39: 746-749.
99. AG Froseth, PM Derlet, HV Swygenhoven (2005) Twinning in Nanocrystalline fcc Metals. Advanced Engineering Materials $7: 16-20$.

100. Harald Rosner, Jurgen Markmann, Jorg Weissmuller (2004) Deformation twinning in nanocrystalline Pd. Philosophical Magazine Letters 84: 321-334.

101. XZ Liao, YH Zhao, SG Srinivasan, YT Zhu (2004) Deformation twinning in nanocrystalline copper at room temperature and low strain rate. Appl Phys Lett 84: 592-594.

102. YM Wang, AM Hodge, J Biener, AV Hamza, DE Barnes (2005) Deformation twinning during nanoindentation of nanocrystalline Ta. Applied Physics Letters 86: 101915.

103. S Ni, YB Wang, $X Z$ Liao, HQ Li, RB Figueiredo, et al. (2011) Effect of grain size on the competition between twinning and detwinning in nanocrystalline metals. Physical Review B 84: 235401.

104. C Ye, A Telang, AS Gill, S Suslov, Y Idell, et al. (2014) Gradient nanostructure and residual stresses induced by Ultrasonic Nano-crystal Surface Modification in 304 austenitic stainless steel for high strength and high ductility. Materials Science \& Engineering A 613: 274-288.

105. ZH Cao, LJ Xu, W Sun, J Shi, MZ Wei, et al. (2015) Size dependence and associated formation mechanism of multiple-fold annealing twins in nanocrystalline $\mathrm{Cu}$. Acta Materialia 95: 312-323.

106. D Bufford, Z Bi, QX Jia, H Wang, X Zhang (2012) Nanotwins and stacking faults in high-strength epitaxial $\mathrm{Ag} / \mathrm{Al}$ multilayer films. Applied Physics Letters 101: 223112.

107. VS Boyko, RYa Kezerashvili (2014) Twinning propensity in nanocrystalline face-centered cubic, body-centered cubic, and hexagonal close-packed metals. Journal of Physics and Chemistry of Solids 75: 1119-1123.

108. VS Boyko, R Ya Kezerashvili (2012) Twinning in nanocrystalline materials. Physics Letters A 376: 1058-1061.

109. P Sharma, S Ganti (2003) On the grain-size-dependent elastic modulus of nanocrystalline materials with and without grain-boundary sliding. Journal of Materials Research 18: $1823-1826$.

110. M Shaat, A Abdelkefi (2016) Reporting buckling strength and elastic properties of nanowires. Journal of Applied Physics 120: 235104.

111. L Tian, I Anderson, T Riedemann, A Russell (2014) Modeling the electrical resistivity of deformation processed metal-metal composites. Acta Materialia 77: 151-161.

112. L Tian, A Russell, T Riedemann, S Mueller, I Anderson (2017) A deformation-processed Al-matrix/Ca-nanofilamentary composite with low density, high strength, and high conductivity. Materials Science and Engineering $A$ 690: 348-354.

113. AF Mayadas, M Shatzkes (1970) Electrical-Resistivity Model for Polycrystalline Films: the Case of Arbitrary Reflection at External Surfaces. Phys Rev B 1: 1382-1389. 